\title{
Protective Effects of Salidroside on Mitochondrial Functions against Exertional Heat Stroke-Induced Organ Damage in the Rat
}

\author{
Wei Zhang, ${ }^{1}$ Ming Peng, ${ }^{2}$ Yang Yang, ${ }^{2}$ Zhangwu Xiao, ${ }^{2}$ Bin Song, ${ }^{3}$ and Zhaofen Lin ${ }^{4}$ \\ ${ }^{1}$ Department of Emergency, The Second Military Medical University Affiliated Fuzhou General Hospital, \\ Fuzhou, Fujian 350025, China \\ ${ }^{2}$ Department of Emergency, Fuzhou General Hospital, Fuzhou, Fujian 350025, China \\ ${ }^{3}$ Medical Department, Fuzhou General Hospital, Fuzhou 350025, China \\ ${ }^{4}$ Emergency Department, Shanghai Changzheng Hospital, Shanghai 200003, China
}

Correspondence should be addressed to Bin Song; gjw1217@163.com and Zhaofen Lin; linzhaofen@sina.com

Received 4 May 2015; Revised 5 July 2015; Accepted 22 July 2015

Academic Editor: Shuang-En Chuang

Copyright $\odot 2015$ Wei Zhang et al. This is an open access article distributed under the Creative Commons Attribution License, which permits unrestricted use, distribution, and reproduction in any medium, provided the original work is properly cited.

Exertional heat stroke (EHS) results in a constellation of systemic inflammatory responses resulting in multiorgan failure and an extremely high mortality. The present study was designed to evaluate the protective effects of salidroside on EHS by improving mitochondrial functions in the rat model. Liver and heart mitochondria were observed by transmission electron microscopy and mitochondrial membrane potential $(\Delta \Psi \mathrm{m})$ was detected by a fluorescent probe. Intramitochondrial free $\mathrm{Ca}^{2+}$ concentration, mitochondrial respiratory control ratio (RCR), reactive oxygen species (ROS) levels, superoxide dismutase (SOD), and malondialdehyde (MDA) activity were detected by the corresponding kits. RT-PCR was performed to estimate peroxisome proliferator-activated receptor- $\gamma$ coactivator- $1 \alpha$ (PGC- $1 \alpha)$ and manganese form of SOD (MnSOD) mRNA expression. The results demonstrated that salidroside was able to relieve EHS damage by reducing the swelling of mitochondria, ROS levels, and MDA activity, as well as increasing $\Delta \Psi \mathrm{m}, \mathrm{RCR}$, free $\mathrm{Ca}^{2+}$ concentration, SOD, PGC- $1 \alpha$, and MnSOD mRNA levels. In conclusion, salidroside has protective effects on mitochondrial functions against exertional heat stroke-induced organ damage in the rat.

\section{Introduction}

There are two types of heat stroke: classic, nonexertional heat stroke (NEHS) and exertional heat stroke (EHS). Classic heat stroke occurs most commonly in very young or older individuals who have health risks and are in poor environmental conditions. Exertional heat stroke occurs more often in younger, healthy individuals who participate in strenuous physical activity. EHS is characterized by hyperthermia, central neurologic disorders, and variable levels of rhabdomyolysis during, or after, an intense or prolonged physical exercise [1]. EHS is a serious disorder that can be fatal especially if proper assessment and treatment are not initiated rapidly [2]. Apart from high temperature, hypoxia and oxidative damage may be responsible for heat stroke. Both high temperature and hypoxia could impair mitochondrial function, aggravate the body's hypoxia, and lead to further deterioration [3]. Heart and liver tissues are tissues with high and fluctuating energy demands, so they were chosen for analysis in this study.

Appropriate regulation of mitochondrial biogenesis and function is a critical component of adaptation to external conditions and prevention of pathogenesis. Mitochondrial dysfunction includes mitochondrial swelling, $\Delta \Psi \mathrm{m}$ decrease, ROS and MDA increase, and antioxidant enzyme SOD reduction [4].

Mitochondria are key regulators of cell death following alterations in $\Delta \Psi \mathrm{m}$ in response to various triggers. Loss of $\Delta \Psi \mathrm{m}$ is a marker of mitochondrial dysfunction that ultimately leads to apoptosis. The respiratory control ratio 
(RCR) is a measure of the "tightness of coupling" between electron transport and oxidative phosphorylation. Deregulation of intraneuronal $\mathrm{Ca}^{2+}$ is known to generate high levels of reactive oxygen species of mitochondrial origin (mtROS), a crucial step in the excitotoxic cascade. An imbalance between production of ROS, oxidative damage to lipids (lipid peroxidation), and its elimination by the antioxidant defense system (such as SOD) in the body has been implicated for causes of aging and mitochondria related disease [4].

Timely protection of mitochondrial function helps to reduce EHS injuries to the body. Salidroside, a phenylpropanoid glycoside isolated from Rhodiola rosea, has been reported to have various pharmacological properties, including attenuating myocardial ischemia-reperfusion injury via PI3K/Akt signaling pathway [5], stabilizing the mitochondrial membrane potential, and inhibiting cell apoptosis by inhibiting hypoxia/hypoglycemia-induced mitochondrial membrane potential damage and reduced activity [6]. In addition, salidroside has been used as an antioxidative therapy for oxidative injury in cardiac diseases and protected against hydrogen peroxide-induced injury in HUVECs via the regulation of REDD1 and mTOR activation [7]. The purpose of this study was to determine whether salidroside could alleviate the symptoms of EHS by protecting mitochondrial functions.

\section{Materials and Methods}

2.1. Ethics Statement. All studies involving animals were carried out according to guidelines put forth by the institutional guidelines for the humane treatment of laboratory animals, as approved under protocol \#IACUC-2012-009 by the Experimental Animal Ethics Committee of Academy of Military Medical Sciences.

2.2. Animals. Male Sprague-Dawley (SD) rats weighing 190$210 \mathrm{~g}$ (8-12-week-old) were obtained from the Animal Center of the Vital River. They were specific pathogen-free animals (SPFA) housed in clean, temperature- and humiditycontrolled SPFA chamber with a room temperature of $22 \pm$ $2^{\circ} \mathrm{C}$, a relative humidity of $50 \pm 5 \%$, unlimited food and water, and a $12 \mathrm{~h}$ light/dark cycle.

2.3. Animal Model and Experimental Design. A total of 80 specific pathogen-free (SPF) SD rats were randomly divided into 8 groups ( $n=10$ per group): control group (Group A), exercise group (Group B), high temperature group (Group C), EHS group (Group D), salidroside low dose group (Group E), salidroside middle dose group (Group F), and salidroside high dose group (Group G). In Group A, rats were fed with normal standard method. In Group B, in the SPF animal room temperature $\left(22^{\circ} \mathrm{C}\right)$, rats were running on a treadmill (treadmill speed: $25 \mathrm{rpm}$ ) and were given a shock (voltage $100 \mathrm{~V}$ ) when they did not run; after the animals rested, they continued to cycle until exhaustion. In Group C, the thermostat inside temperature was adjusted to $45^{\circ} \mathrm{C}$; rats do not exercise until exhaustion. In Group D, the thermostat inside temperature was adjusted to $45^{\circ} \mathrm{C}$, with rats running according to the first set of Group B. Rats only received salidroside in the absence of heat or exercise (Group H, $n=$ 10) to explore whether the effect of salidroside was specific to heat stroke or a nonspecific effect that causes $\mathrm{Ca}^{2+}$ increase and other responses regardless of whether one has heat stroke or not. The animals were sacrificed and the tissues were taken when they stopped running. Salidroside (with different doses, $4 \mathrm{mg} / \mathrm{kg}, 10 \mathrm{mg} / \mathrm{kg}$, and $25 \mathrm{mg} / \mathrm{kg}$, resp.) was administered into rats by gavage [8].

2.4. Mitochondria Isolation. Mitochondria of rat's heart and liver tissues were isolated using a mitochondria isolation kit according to the manufacturer's instructions (Pierce, Rockford, IL, USA) [9]. Briefly, samples were pelleted at $850 \times \mathrm{g}$ for $2 \mathrm{~min}$ by centrifugation before mitochondria isolation reagents $\mathrm{A}, \mathrm{B}$, and $\mathrm{C}$ were added in the correct sequence. The mixture was centrifuged at $700 \times \mathrm{g}$ for $10 \mathrm{~min}$ at $4^{\circ} \mathrm{C}$. The supernatant was transferred to a new, $2.0 \mathrm{~mL}$ tube and centrifuged at $12,000 \mathrm{~g}$ for $15 \mathrm{~min}$ at $4^{\circ} \mathrm{C}$. Mitochondria isolation reagent $C$ was added to the pellet and centrifuged at $12,000 \times \mathrm{g}$ for $5 \mathrm{~min}$ before the pellet was resuspended in $80 \mathrm{mmol} / \mathrm{L}$ sucrose with $0.1 \%$ bovine serum albumin (BSA). Mitochondrial fractions were kept at $4^{\circ} \mathrm{C}$ and studied within $3 \mathrm{~h}$ of isolation. A protein content assay (Bio-Rad, Hercules, CA, USA) was performed on the isolated mitochondria fractions.

2.5. Transmission Electron Microscopic Observation. Electron photomicrographs for hepatocytes and cardiomyocytes were prepared as described [9]. In brief, cells were washed with PBS and fixed in 4\% glutaraldehyde/1\% paraformaldehyde for $2 \mathrm{~h}$ at room temperature and postfixed in $1 \% \mathrm{OsO}_{4}$ for $1 \mathrm{~h}$ at $4^{\circ} \mathrm{C}$. After dehydration in graded ethanols $(70 \%, 95 \%$, and 2 $\times 100 \%$ ), cells were embedded in LX112. Ultrathin sections were stained with $1 \%$ uranyl acetate and lead citrate and then were examined for mitochondrial morphology on a H7000 electron microscope operating at $80 \mathrm{kV}$ (Hitachi) [10].

2.6. Mitochondrial Membrane Potential Assay. Mitochondrial membrane potential $(\Delta \Psi \mathrm{m})$ was detected using a fluorescent probe, tetramethylrhodamine methyl ester (TMRM; Molecular Probes Inc., Leiden, Netherlands), which reversibly accumulates according to the membrane potential. Briefly, isolated hepatocytes and cardiomyocytes were incubated in HEPES buffer $(134 \mathrm{mmol} / \mathrm{L} \mathrm{NaCl}, 6 \mathrm{mmol} / \mathrm{L}$ $\mathrm{KCl}, 2 \mathrm{mmol} / \mathrm{L} \mathrm{CaCl}, 1 \mathrm{mmol} / \mathrm{L} \mathrm{MgCl}_{2}, 10 \mathrm{mmol} / \mathrm{L}$ HEPES, and $10 \mathrm{mmol} / \mathrm{L}$ glucose; $\mathrm{pH}$ 7.40) containing TMRM (100 nmol/L) for $30 \mathrm{~min}$ followed by a 15 -minute wash. Flow cytometric analysis (BD FACSCalibur) was performed to analyze mean fluorescence intensity (MFI) [11].

2.7. Mitochondrial Respiratory Control Ratio. A commercially available kit (Genmed Scientifics Inc., Wilmington, DE, USA) was used to investigate mitochondrial respiratory control ratio (RCR) according to the manufacturer's instructions. The respiratory control ratio (RCR) was determined by calculating the ratio of State 3 to State 4 . The RCR is a general measure of mitochondrial function and higher values denote better function. State 4 respiration is a model of steady-state, basal 
activity, while State 3 respiration is measured following the addition of ADP and mimics mitochondrial activity during exercise with high oxygen consumption and ATP turnover. The RCR was measured at $25^{\circ} \mathrm{C}$. Briefly, mitochondrial fractions were incubated with Genmed reagent A. State 4 substrate solution (reagent B) and State 3 substrate solution (reagent C) were added in the proper sequence. Mitochondrial State 3 and State 4 respiration rates were determined and mitochondrial RCR was calculated as follows: $\mathrm{RCR}=$ State 3 respiration rate/State 4 respiration rate [12].

\subsection{Measurements of the Intramitochondrial Free $\mathrm{Ca}^{2+}$ Con-} centration. The high-affinity cell-permeant $\mathrm{Ca}^{2+}$ indicator Fluo-4AM (Molecular Probes) was excited using short (about 1s) flashes of light at $488 \pm 10 \mathrm{nM}$ and its emission was recorded using Flow Cytometry (BD FACS). Fluo-4AM was dissolved in DMSO and stored frozen (no more than a week) in the dark. Prior to the experiment, Pluronic F-127 was added to facilitate solution of Fluo-4AM in the aqueous (physiological) solution. In the working solution the final concentration of Fluo-4AM was $1 \mu \mathrm{M}$, whereas the contents of DMSO and Pluronic F-127 were no more than $0.001 \%$. The isolated preparation was stained with $1 \mu \mathrm{M}$ Fluo-4AM for $10 \mathrm{~min}$ at room temperature. Afterwards, the specimen was perfused with a physiological solution for $40 \mathrm{~min}$, and measurements of the fluorescence were performed. Flow cytometric analysis (BD FACSCalibur) was performed to analyze mean fluorescence intensity (MFI).

\subsection{Mitochondrial Reactive Oxygen Species Assay.} H2DCFDA $\left(2^{\prime}, 7^{\prime}\right.$-dichlorodihydrofluorescein diacetate, Molecular Probes) was used as a cell-permeant indicator for ROS. The dye was excited using a $1 \mathrm{~s}$ flash of light at 480/ $15 \mathrm{~nm}$ every minute and its emission was recorded using Flow Cytometry (BD FACS). H2DCFDA was dissolved in DMSO (dimethyl sulfoxide). Pluronic F-127 (Molecular Probes) was used to enhance solution of the dye. The final concentration of DMSO and Pluronic F-127 in the working solution did not exceed $0.001 \%$. The preparation was incubated with H2DCFDA $(2 \mu \mathrm{M})$ at room temperature for 15 min and after that it was perfused with physiological saline for $45 \mathrm{~min}$ before recording fluorescence intensity [13]. Flow cytometric analysis (BD FACSCalibur) was performed to analyze mean fluorescence intensity (DCF).

2.10. Mitochondrial Lipid Peroxidation Assay. The SOD activity was determined using SOD Assay Kit (Sigma-Aldrich, St. Louis, MO, USA). According to the provided protocol, the assay was based on utilization of Dojindo's highly water-soluble tetrazolium salt, WST-12-(4-iodophenyl)-3(4-nitrophenyl)-5-(2,4-disulfophenyl)-2H-tetrazolium, monosodium salt, which produced a water-soluble formazan dye upon reduction with a superoxide anion. The rate of the reduction with $\mathrm{O}_{2}$ is linearly related to xanthine oxidase (XO) activity and is inhibited by SOD. The SOD activity as an inhibition activity was quantified by measuring the decrease in the color development at $450 \mathrm{~nm}$. The SOD activity in the plasma was expressed as $\mathrm{U} / \mathrm{mg}$ protein [14]. MDA is a sensitive marker of lipid peroxidation. A mitochondrial MDA assay was performed using a commercially available kit (Calbiochem, San Diego, CA, USA) according to the manufacturer's instructions. Briefly, mitochondrial fractions were resuspended in $5 \mathrm{mmol} / \mathrm{L}$ butylated hydroxytoluene. For each reaction, $200 \mu \mathrm{L}$ of mitochondria sample or standard was added to $650 \mu \mathrm{L}$ chromogenic reagents and $150 \mu \mathrm{L}$ of $12 \mathrm{~N} \mathrm{HCl}$ at $45^{\circ} \mathrm{C}$ for $60 \mathrm{~min}$. Samples were then centrifuged at $10,000 \times \mathrm{g}$ for $5 \mathrm{~min}$ at $4^{\circ} \mathrm{C}$ and the supernatant was collected. The absorbance at $525 \mathrm{~nm}$ was recorded. The level of mitochondrial MDA was calculated using a standard curve.

\subsection{Quantitative Reverse Transcription-Polymerase Chain} Reaction. To quantitatively estimate PGC- $1 \alpha$ and MnSOD mRNA expression in the rat's hepatocytes and cardiomyocytes, real-time polymerase chain reaction (RT-PCR) was performed using the following primer pair: PGC$1 \alpha$ forward primer $5^{\prime}$-CACCAAACCCACAGAGAACAG$3^{\prime}$ and PGC- $1 \alpha$ reverse primer $5^{\prime}$-GCAGTTCCAGAGAGTTCCACA- $3^{\prime}$; MnSOD forward primer $5^{\prime}$-CCAAGGGAGATGTTACAACTCAG-3' and MnSOD reverse primer $5^{\prime}$-GGGCTCAGGTTTGTCCAGAA-3'. We used glyceraldehyde-3phosphate dehydrogenase (GAPDH) as the internal control: GAPDH forward primer $5^{\prime}$-TGGTATCGTGGAAGGACTCATGAC- $3^{\prime}$ and GAPDH reverse primer $5^{\prime}$-ATGCCAGTGAGCTTCCCGTTCAGC- $3^{\prime}$. RNA isolation and conversion to complementary DNA (cDNA) were performed using a RevertAid First Strand cDNA Synthesis Kit (\#K1622, Fermentas Inc., Hanover, MD, USA) following the manufacturer's instructions. cDNA suspension $(1 \mu \mathrm{g})$ was amplified with SYBR Green Quantitative PCR Master Mix (Applied Biosystems, Foster City, CA, USA). Assays were performed in triplicate with the ABI7500 instrument. The quantitative PGC- $1 \alpha$ and MnSOD values for each cell line were estimated by dividing them by the GAPDH expression levels.

2.12. Statistical Analysis. Results are expressed as mean \pm SEM. Two-way ANOVA was performed using SPSS17.0. Tests of equality of variance were carried out and post hoc tests were also performed. $P$ value $<0.05$ was considered statistically significant; ${ }^{*} P<0.05 ;{ }^{* *} P<0.01 ;{ }^{* * *} P<0.001$.

\section{Results}

3.1. Salidroside Significantly Reduced the Mitochondrial Lesions. The animals were sacrificed and the tissues were taken when they stopped running [15]. There was water during heating. Table 1 showed that the rats exercising in the heat suffered exertional heat stroke at exhaustion. The body temperature of the rats at that time was $46.2 \pm 3.7^{\circ} \mathrm{C}$ in the EHS group rats. High temperature and exercise caused morphology changes of mitochondrial inner structure. As displayed in Figure 1, cardiomyocytes from EHS group rats were swelling with mitochondrial myelin breakdown and disappearance of cristae matrix granules. Also, electronlucent areas were formed in mitochondrial matrix or concentric cristae $(15,000 x)$. Compared to the EHS group, 
TABle 1: Physical parameters of the rats.

\begin{tabular}{lccr}
\hline Group & Time to exhaustion $(\mathrm{min})$ & How far they ran $(\mathrm{m})$ & The body temperature \\
\hline Control & $45.3 \pm 1.1$ & $203.4 \pm 3.2$ & $38.2 \pm 1.3^{\circ} \mathrm{C}$ \\
Salidroside & $42.1 \pm 2.3$ & $198.2 \pm 2.1$ & $38.5 \pm 0.9^{\circ} \mathrm{C}$ \\
Exercise & $35.3 \pm 3.4$ & $167.3 \pm 1.1$ & $42.7 \pm 0.6^{\circ} \mathrm{C}$ \\
High temperature & $36.2 \pm 1.2$ & $158.9 \pm 2.9$ & $43.4 \pm 2.3^{\circ} \mathrm{C}$ \\
EHS & $28.3 \pm 2.3$ & $129.2 \pm 1.2$ & $46.2 \pm 3.7^{\circ} \mathrm{C}$ \\
Low dose & $34.8 \pm 2.1$ & $147.8 \pm 3.8$ & $44.2 \pm 1.7^{\circ} \mathrm{C}$ \\
Middle dose & $38.9 \pm 2.8$ & $167.2 \pm 2.7$ & $40.5 \pm 1.3^{\circ} \mathrm{C}$ \\
High dose & $41.2 \pm 3.2$ & $188.3 \pm 1.8$ & $39.5 \pm 0.7^{\circ} \mathrm{C}$ \\
\hline
\end{tabular}
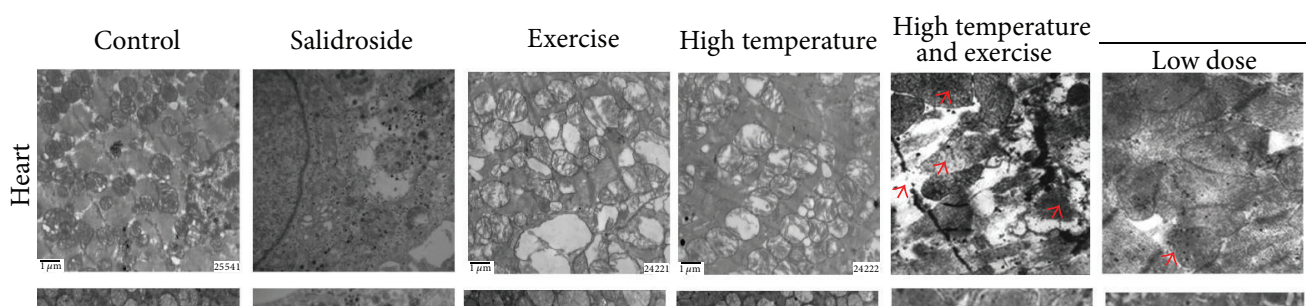

Salidroside
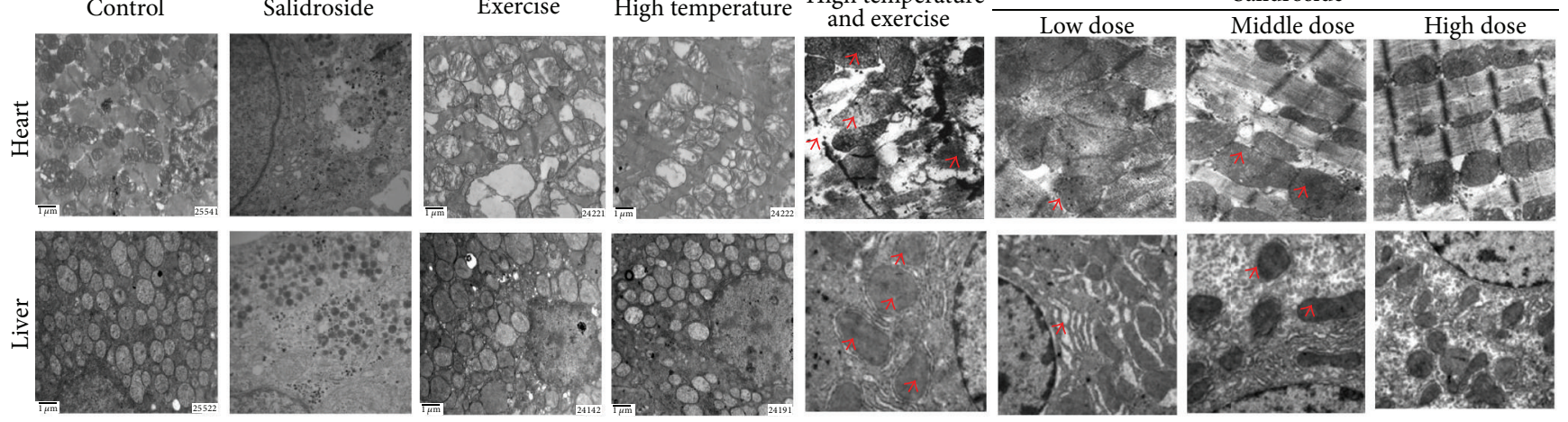

FIGURE 1: Salidroside suppressed EHS-induced morphologic alterations of mitochondria in cardiomyocytes and hepatocytes. Mitochondrial morphology in the EHS group and EHS + salidroside group $(20,50$, and $100 \mu \mathrm{M})$ was observed by an electron microscope $(15,000 \mathrm{x})$. The red arrowheads indicated the mitochondrial swelling, myelin breakdown, and disappearance of cristae matrix granules and, in mitochondrial matrix or concentric cristae, indicated the electron-lucent areas formed.

mitochondrial lesions were attenuated in rats treated with salidroside at different concentrations $(20,50$, and $100 \mu \mathrm{M})$. Similar results were also obtained from hepatocytes.

\subsection{Salidroside Restored EHS-Induced Decrease in $\Delta \Psi m$.} Mitochondria are key regulators of cell death following alterations in $\Delta \Psi \mathrm{m}$ in response to various triggers. $\Delta \Psi \mathrm{m}$ in hepatocytes and cardiomyocytes was determined after treatment with high temperature, exercise, and/or various concentrations of salidroside $(20,50$, and $100 \mu \mathrm{M})$. A $79.4 \pm$ $5.5 \%$ reduction in average fluorescent intensity incorporated into hepatocytes mitochondria was detected in EHS group $($ EHS group $=173.5 \pm 7.8$ versus control group $=788.5 \pm 10.1$, $P<0.001$, Figure 2(b)) when compared to the control group. Consistent results were also obtained from cardiomyocytes with a $73.4 \pm 6.7 \%$ reduction (EHS group $=183.0 \pm 9.8$ versus control group $=635.5 \pm 6.6, P<0.001$, Figure 2(c)). $\Delta \Psi \mathrm{m}$ was gradually increased after treatment with different concentrations of salidroside. After treatment with $100 \mu \mathrm{M}$ salidroside, $\Delta \Psi \mathrm{m}$ in hepatocytes increased $71.6 \pm 5.2 \%$ compared to the EHS group (salidroside group $=621.5 \pm 6.3$ versus EHS group $=173.5 \pm 7.8, P<0.001$, Figure $2(b)$ ). Similar results were also obtained from cardiomyocytes with a $69.9 \pm 4.8 \%$ increase (Figure $2(\mathrm{c})$ ).

3.3. Salidroside Improved RCR Decreased in EHS Rats. The respiratory control ratio (RCR), a measure of the "tightness of coupling" between electron transport and oxidative phosphorylation, was determined from the ratio of State 3 to State 4 rates of respiration. In hepatocytes, the RCR was significantly decreased in exercise group, high temperature group, or EHS group compared with the control group (exercise group = $4.4 \pm 0.3$, high temperature group $=2.9 \pm 0.4$, and $\mathrm{EHS}$ group $=$ $1.8 \pm 0.1$ versus control group $=7.8 \pm 0.5$, all $P<0.001$, Figure 3(a)). However, the RCR was gradually improved in the salidroside groups with the increase of concentration when compared to EHS group (middle dose group $=4.0 \pm 0.4$ and high dose group $=6.4 \pm 0.2$ versus EHS group $=1.8 \pm$ 0.1 , all $P<0.001$, Figure 3(a)). Consistent results were also obtained from cardiomyocytes (Figure 3(b)).

3.4. Salidroside Restored EHS-Triggered Decrease in $\mathrm{Ca}^{2+}$ Concentration. $\mathrm{Ca}^{2+}$ concentrations were measured after treatment with high temperature, exercise, and/or various concentrations of salidroside $(20,50$, and $100 \mu \mathrm{M})$. Compared with the control group, a $53.4 \pm 3.5 \%$ reduction in average fluorescent intensity incorporated into hepatocytes mitochondria was detected in EHS group (EHS group $=27.3 \pm$ 2.1 versus control group $=53.0 \pm 2.8, P<0.001$, Figure $4(\mathrm{~b})$ ). Similarly, the average fluorescent intensity incorporated into cardiomyocytes mitochondria was reduced $36.1 \pm 1.3 \%$ in EHS group (EHS group $=38.1 \pm 4.3$ versus control group $=$ $62.2 \pm 3.1, P<0.05$, Figure $4(\mathrm{c})) . \mathrm{Ca}^{2+}$ concentration was 

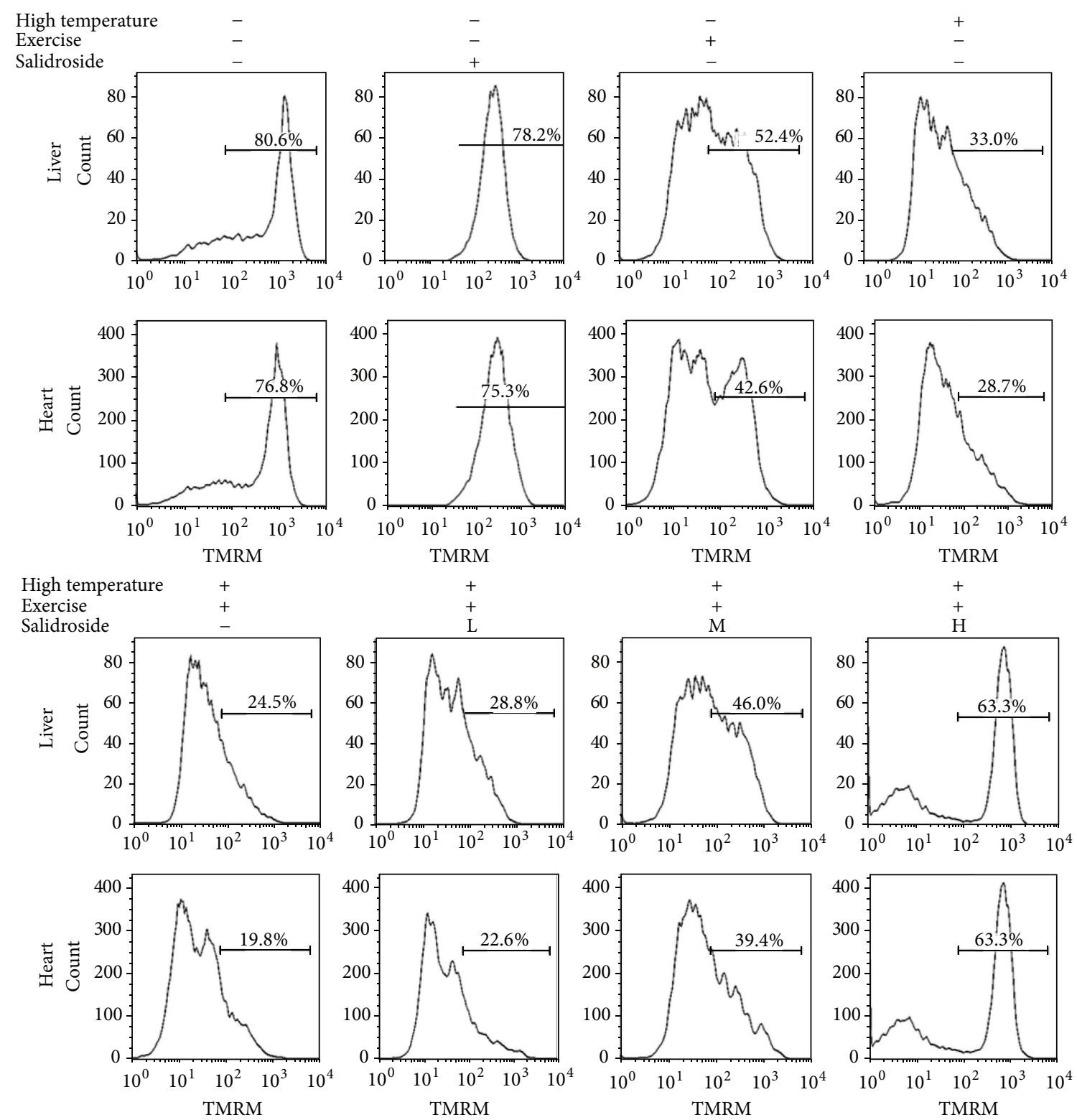

(a)

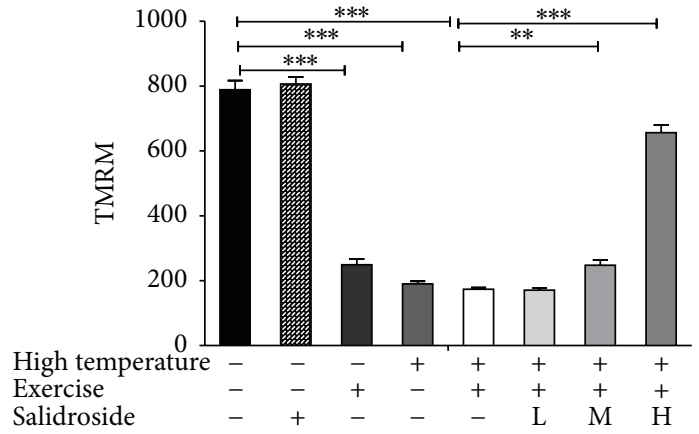

(b)

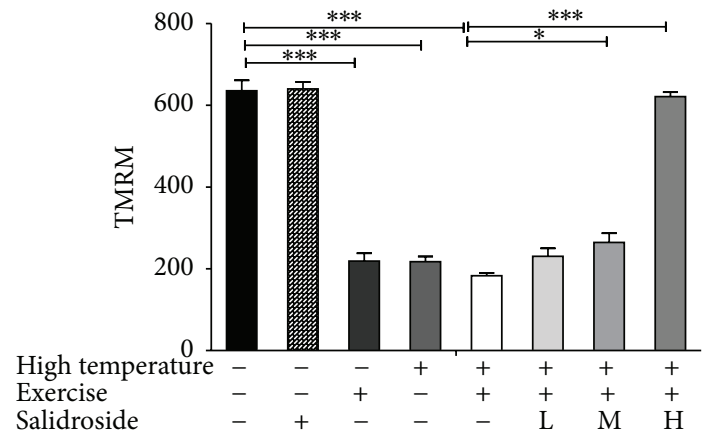

(c)

FiguRE 2: Salidroside restored EHS-induced decrease in $\Delta \Psi \mathrm{m}$. (a) $\Delta \Psi \mathrm{m}$ (reflected by tetramethylrhodamine methyl ester) was assayed in hepatocytes and cardiomyocytes in the control group, high temperature group, exercise group, EHS group, and EHS + salidroside group (20, 50, and $100 \mu \mathrm{M}$ ). (b) $\Delta \Psi \mathrm{m}$ in hepatocytes in the control group, high temperature group, exercise group, EHS group, and EHS + salidroside group $(20,50$, and $100 \mu \mathrm{M})$. (c) $\Delta \Psi \mathrm{m}$ in cardiomyocytes in the control group, high temperature group, exercise group, EHS group, and EHS + salidroside group $(20,50$, and $100 \mu \mathrm{M})$. Results were expressed as the mean \pm SEM. $n=10 .{ }^{*} P<0.05,{ }^{* *} P<0.01$, and ${ }^{* * *} P<0.001$. 


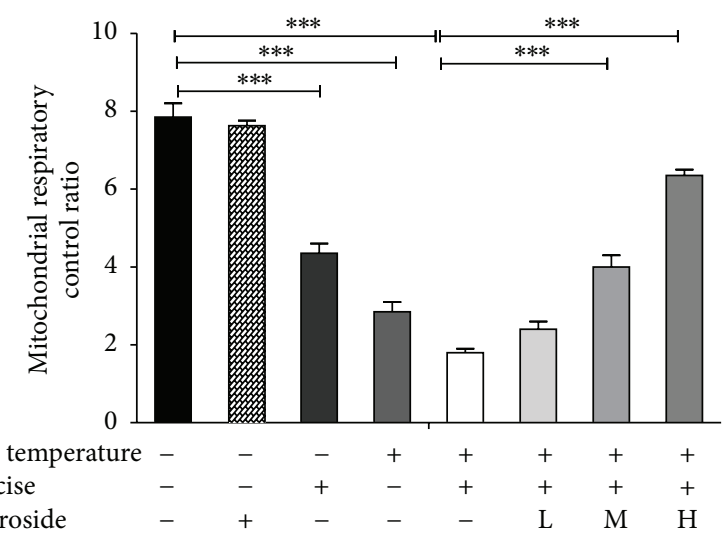

(a)

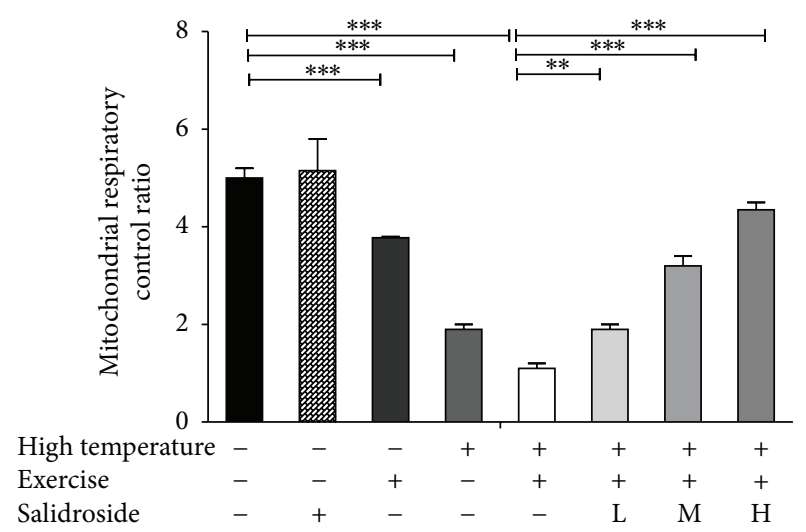

(b)

FIGURE 3: Salidroside increased the RCR. (a) RCR detected in hepatocytes in the control group, high temperature group, exercise group, EHS group, and EHS + salidroside group (20,50, and $100 \mu \mathrm{M})$. (b) RCR detected in cardiomyocytes in the control group, high temperature group, exercise group, EHS group, and EHS + salidroside group $(20,50$, and $100 \mu \mathrm{M})$. Results were expressed as the mean \pm SEM. $n=10 .{ }^{* *} P<0.01$, and ${ }^{* * *} P<0.001$.

gradually increased after treatment with different concentrations of salidroside. After treatment with $100 \mu \mathrm{M}$ salidroside, $\mathrm{Ca}^{2+}$ concentration in hepatocytes increased $71.6 \pm 5.2 \%$ compared to the EHS group (salidroside group $=57.9 \pm 3.3$ versus EHS group $=27.3 \pm 2.1, P<0.001$, Figure 4(b)) Similar results were also obtained from cardiomyocytes with a $23.2 \pm 1.6 \%$ reduction (Figure $4(\mathrm{c})$ ).

\subsection{Salidroside Reduced ROS Levels Caused by EHS Condition.} To investigate the effects of salidroside on EHS, ROS levels in hepatocytes and cardiomyocytes were evaluated using specific fluorescent probes for ROS. ROS production in hepatocytes was increased significantly in EHS group compared to that of the control group (EHS group $=112.3 \pm 1.7$ versus control group $=46.2 \pm 0.9, P<0.001$, Figure 5(b)). However, the ROS levels were significantly reduced in salidroside groups (salidroside group $=43.9 \pm 1.4$ versus EHS group $=$ $112.3 \pm 1.7, P<0.01$, Figure $5(\mathrm{~b}))$. Similar results were also obtained from cardiomyocytes (Figure 5(c)).

3.6. Salidroside Inhibited EHS-Induced MDA Activity Increase and Enhanced EHS-Triggered SOD Activity Decrease. The activity of SOD decreased significantly in EHS group compared to that of the control group $(P<0.001)$. A significant decrease in SOD activity was also observed in the high temperature and exercise groups $(P<0.05$ and $P<0.001$, resp.). SOD activity was gradually increased after treatment with different concentrations of salidroside (Figures 6(a) and 6(b)). However, MDA activity was increased significantly in salidroside groups when compared to the EHS group. Increased endogenous MDA and decreased SOD indicated that the balance changed on the behalf of prooxidation in the liver homogenates of EHS rats.

3.7. Upregulation of PGC-1 $\alpha$ and MnSOD in EHS Rats by Salidroside. PGC- $1 \alpha$ and MnSOD levels were assayed by RTPCR method. The results showed the PGC- $1 \alpha$ mRNA and
MnSOD mRNA levels in hepatocytes and cardiomyocytes were downregulated in EHS group when compared to control group (Figure 7). However, the level of PGC- $1 \alpha$ mRNA and MnSOD activity was increased significantly in salidroside groups when compared to the EHS group. This was consistent with recent data in that mitochondrial damage and PGC$1 \alpha$ downregulation are key events in MAO-A-dependent cardiomyocyte necrosis [16].

\section{Discussion}

EHS is a kind of severe illness characterized by central nervous system (CNS) abnormalities and potentially tissue damage resulting from elevated body temperatures induced by strenuous physical exercise and increased environmental heat stress. The high mortality rate of EHS is usually associated with multiorgan failure, especially coagulopathy and hepatic injury [17]. Both high temperature and hypoxia could impair mitochondrial function. The heart and liver are very susceptible to acute hypoxia or ischemia, because they are organs with high oxygen consumption [18]. Mitochondrial dysfunction included mitochondria swelling, $\Delta \Psi \mathrm{m}$ decrease, ROS and MDA increase, and antioxidant enzyme SOD reduction. Appropriate regulation of mitochondrial biogenesis and function is a critical component of adaptation to external conditions and prevention of pathogenesis [19]. Salidroside has been demonstrated to have multiple biological effects [1922]. In this study, we demonstrate that preservation of mitochondria function is the major mechanism for salidroside against EHS.

Loss of $\Delta \Psi \mathrm{m}$ is a marker of mitochondrial dysfunction that ultimately leads to apoptosis. Mitochondria are key regulators of cell death following alterations in $\Delta \Psi \mathrm{m}$ in response to various triggers. Our results demonstrated that $\Delta \Psi \mathrm{m}$ reduced significantly in EHS rats, which is consistent with previous report which demonstrated that depletion of $\Delta \Psi \mathrm{m}$ in EHS rats was early marker of the apoptotic 

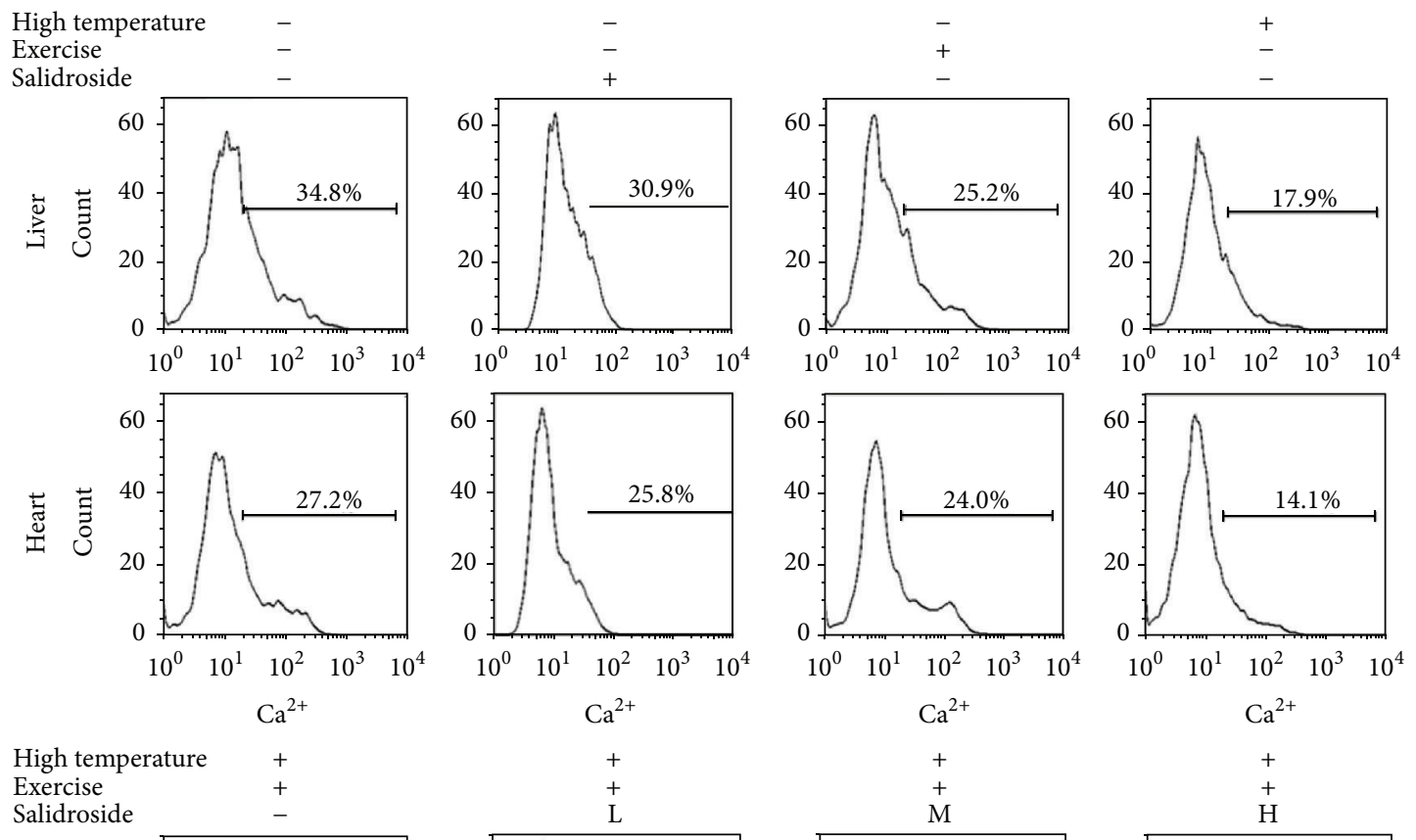

Salidroside
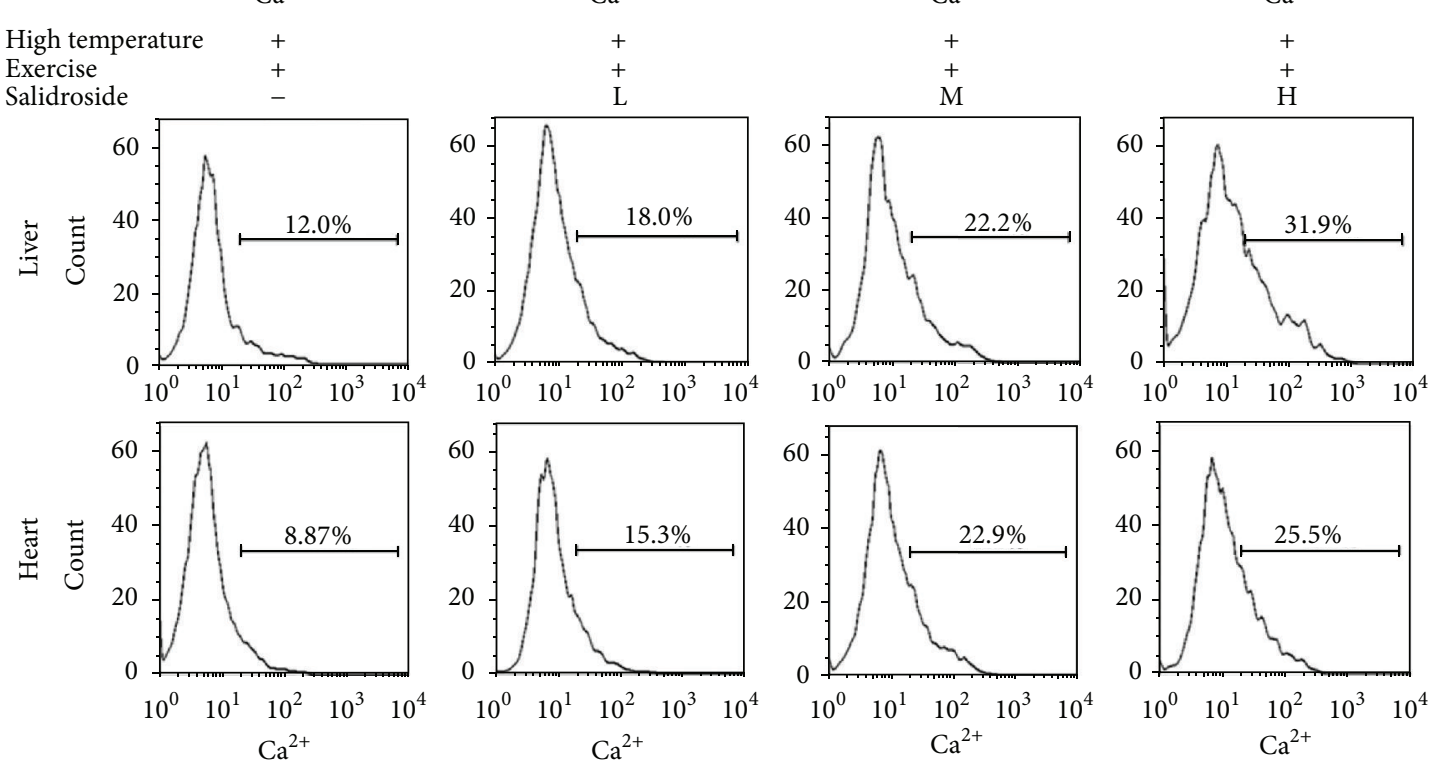

(a)

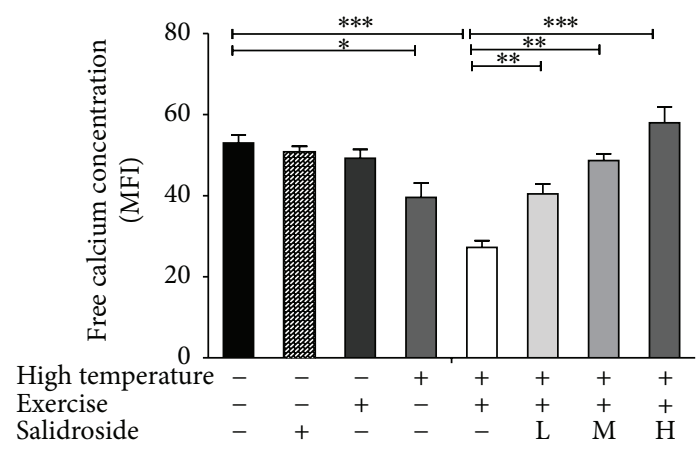

(b)

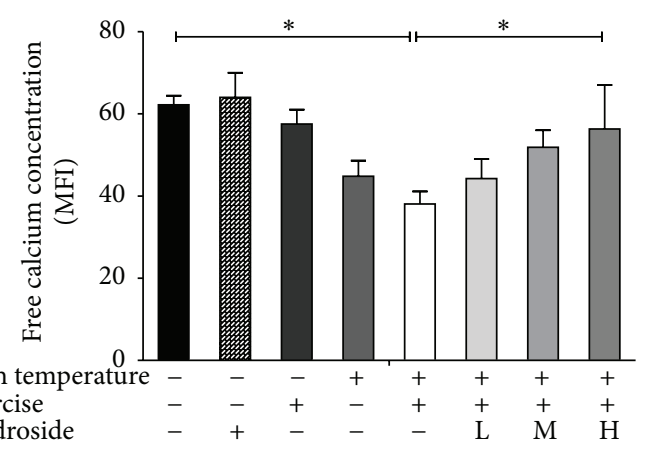

(c)

FIGURE 4: Salidroside restored EHS-triggered decrease in $\mathrm{Ca}^{2+}$ concentration. (a) $\mathrm{Ca}^{2+}$ concentration was detected by inverted fluorescence microscope in hepatocytes and cardiomyocytes in the control group, high temperature group, exercise group, EHS group, and EHS + salidroside group $(20,50$, and $100 \mu \mathrm{M})$. (b) Free $\mathrm{Ca}^{2+}$ concentration in hepatocytes in the control group, high temperature group, exercise group, EHS group, and EHS + salidroside group (20, 50, and $100 \mu \mathrm{M})$. (c) Free $\mathrm{Ca}^{2+}$ concentration in cardiomyocytes in the control group, high temperature group, exercise group, EHS group, and EHS + salidroside group (20, 50, and $100 \mu \mathrm{M})$. Results were expressed as the mean \pm SEM. $n=10 .{ }^{*} P<0.05,{ }^{* *} P<0.01$, and ${ }^{* * *} P<0.001$. 

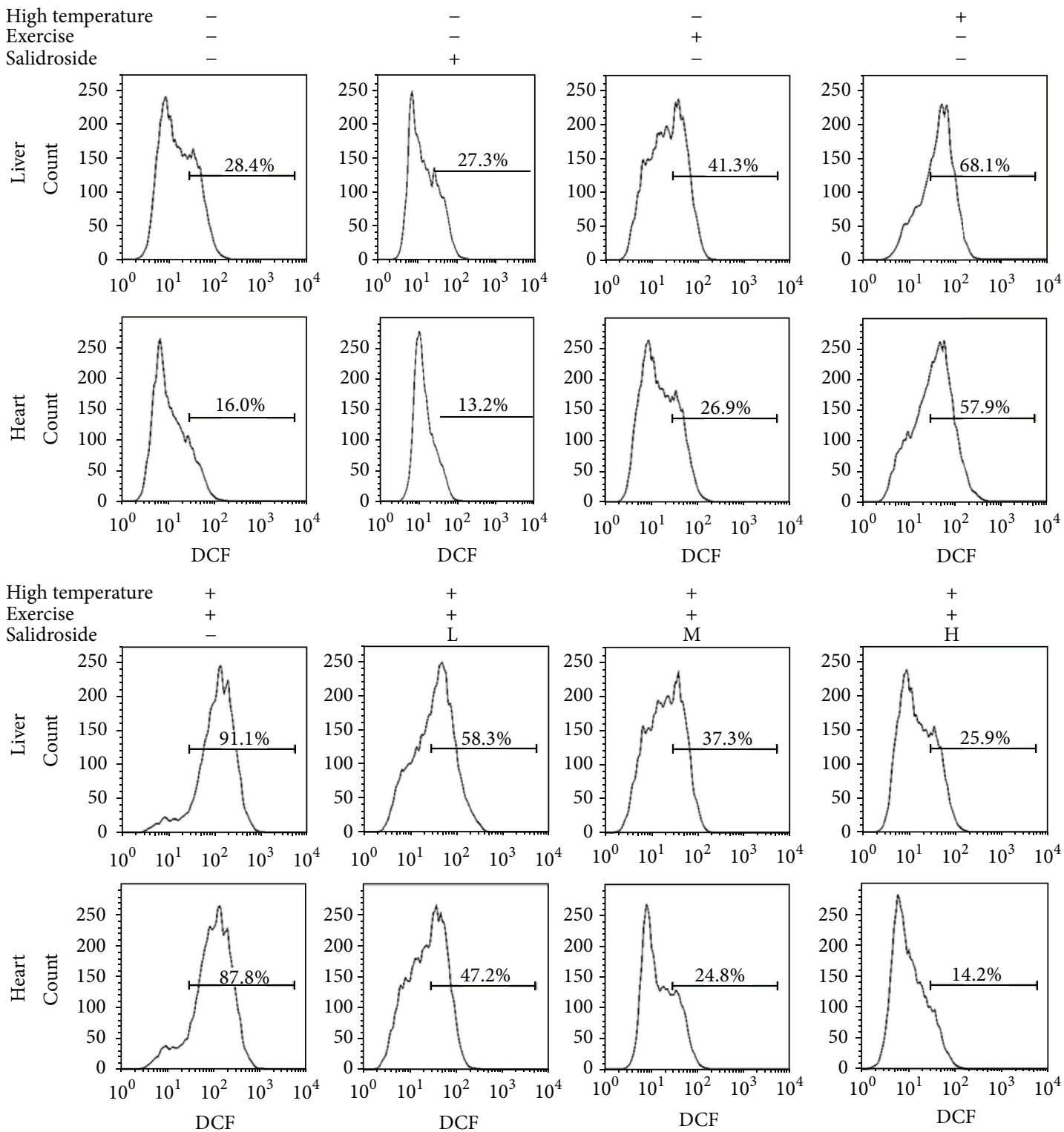

(a)

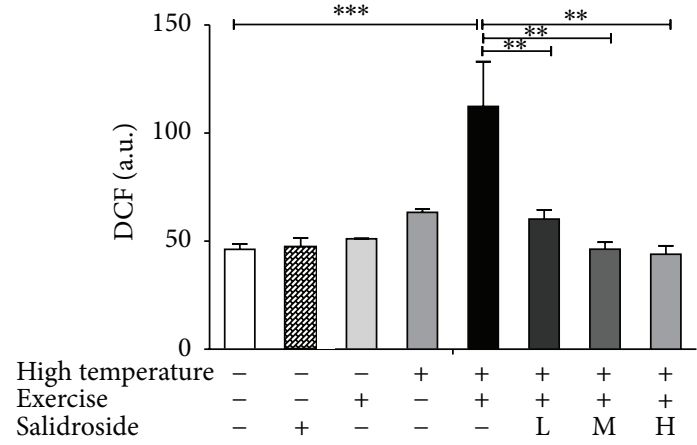

(b)

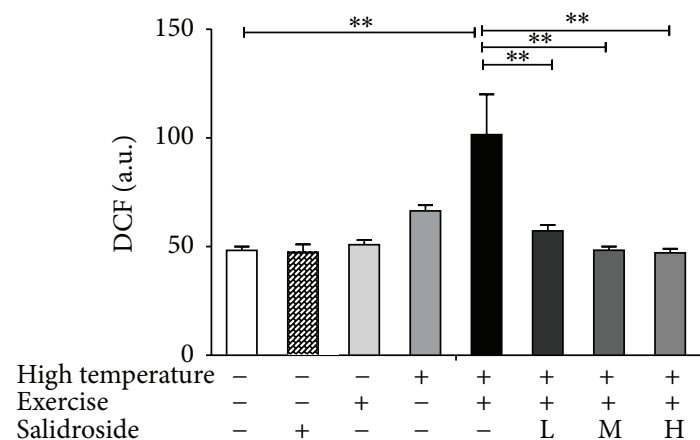

(c)

FIGURE 5: Salidroside inhibited excessive generation of ROS triggered by EHS. (a) ROS detected using specific fluorescent probes in hepatocytes and cardiomyocytes in the control group, high temperature group, exercise group, EHS group, and EHS + salidroside group $(20,50$, and $100 \mu \mathrm{M})$. (b) ROS level in hepatocytes in the control group, high temperature group, exercise group, EHS group, and EHS + salidroside group $(20,50$, and $100 \mu \mathrm{M})$. (c) ROS level in cardiomyocytes in the control group, high temperature group, exercise group, EHS group, and EHS + salidroside group $(20,50$, and $100 \mu \mathrm{M})$. Results were expressed as the mean \pm SEM. $n=10 .{ }^{* *} P<0.01$, and ${ }^{* * *} P<0.001$. 


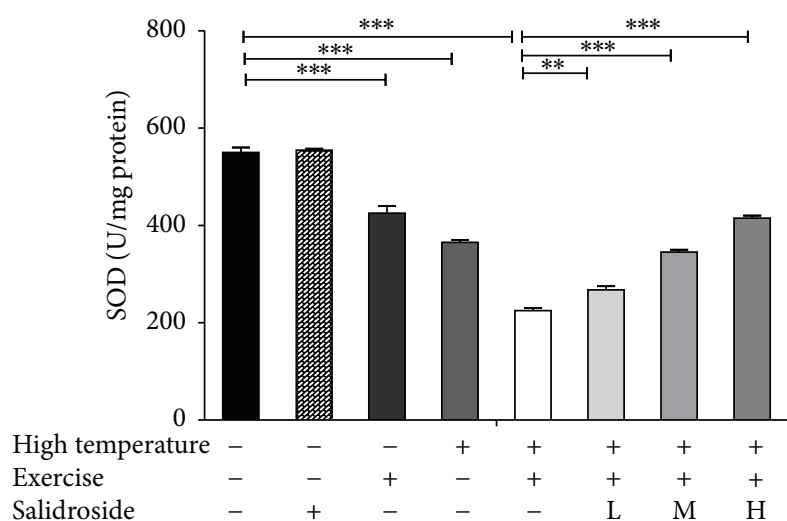

(a)

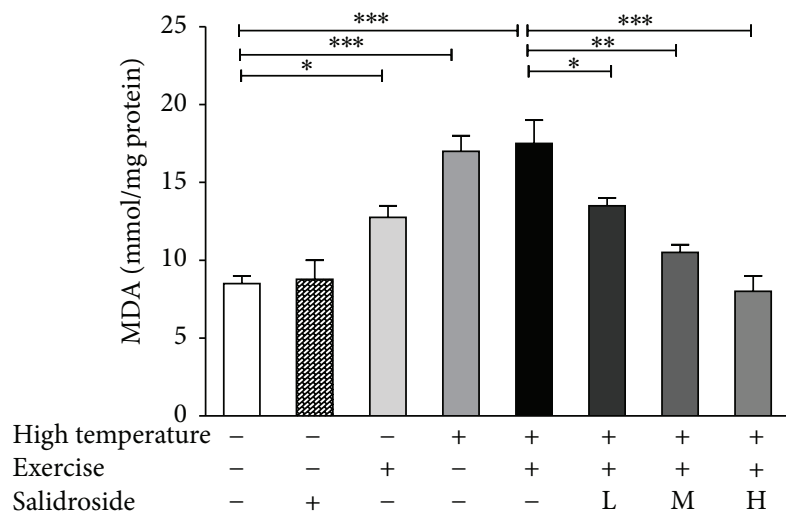

(c)

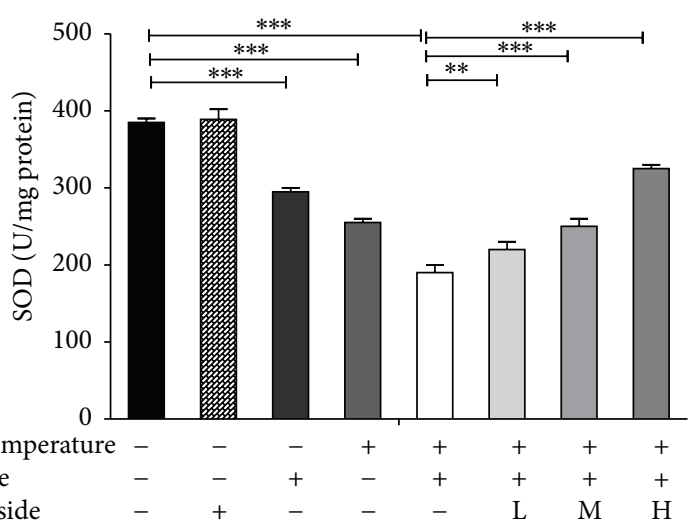

(b)

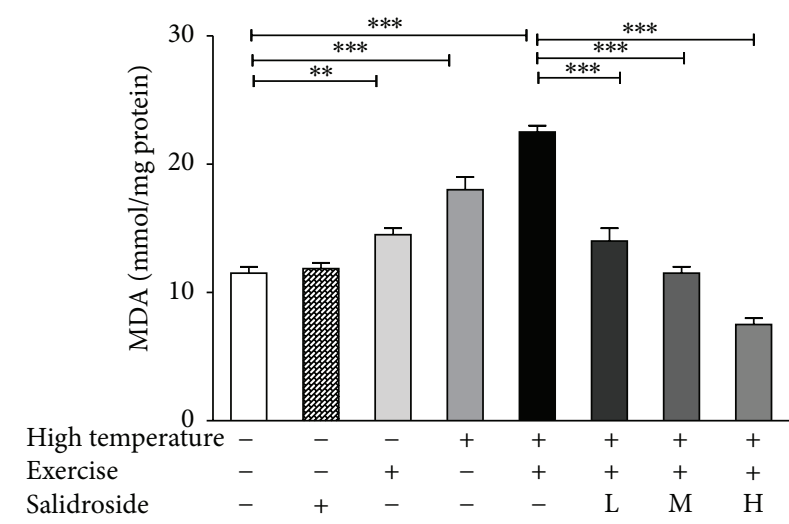

(d)

FIGURE 6: Salidroside inhibited EHS-induced MDA activity increase and enhanced EHS-triggered SOD activity decrease. (a) SOD activities in hepatocytes in the control group, high temperature group, exercise group, EHS group, and EHS + salidroside group (20, 50, and $100 \mu \mathrm{M})$. (b) SOD activities in cardiomyocytes in distinct treatment groups. (c) MDA levels in hepatocytes in distinct treatment groups. (d) MDA levels in hepatocytes in distinct treatment groups. Results were expressed as the mean \pm SEM. $n=10 .{ }^{*} P<0.05,{ }^{* *} P<0.01$, and ${ }^{* * *} P<0.001$.

process [18]. Moreover, we found that salidroside restored the depolarization of $\Delta \Psi \mathrm{m}$ induced by EHS.

Our results showed that EHS caused significantly reduced $\mathrm{Ca}^{2+}$ concentration in hepatocytes and cardiomyocytes but induced a rapid and potent increase in ROS. Deregulation of intraneuronal $\mathrm{Ca}^{2+}$ is known to generate high levels of reactive oxygen species of mitochondrial origin (mt-ROS), a crucial step in the excitotoxic cascade [21]. The consequential role of ROS in the mechanism of cell cytotoxicity, especially the induction of apoptosis, has received increased attention in the field of EHS [22]. Salidroside was able to increase free $\mathrm{Ca}^{2+}$ concentration and reduce ROS production, thus relieving EHS damage.

An imbalance between production of ROS, oxidative damage to lipids (lipid peroxidation), and its elimination by the antioxidant defense system (such as SOD) in the body has been implicated for causes of aging and mitochondria related diseases [23]. Oxidative stress due to exhaustive training induced ROS increase in red gastrocnemius muscles, which led to a decrease in the cell antiapoptotic ability. Salidroside dramatically inhibited excessive generation of ROS by enhancing SOD activity, therefore protecting cardiomyocytes and hepatocytes against EHS-generated ROS attack, in accordance with the effect of salidroside to preserve, unaltered the mitochondrial ultrastructure as revealed by electron microscopy images. MDA, a product of lipid peroxidation, is elevated after EHS induction.

Moreover, we found that salidroside significantly enhanced SOD activity, while MDA activity was significantly decreased. These results suggested that the cardioprotective and hepatoprotective effects of salidroside might be related to the inhibition of ROS overgeneration and predisposing preservation of intact cells and integrity of mitochondria. PGC- $1 \alpha$ is a transcriptional master coregulator of mitochondrial biogenesis, metabolism, and antioxidant defenses [24]. PGC-1 $\alpha$, which has been demonstrated to produce more uncoupled mitochondria, appears to have a stronger influence over antioxidant proteins, such as MnSOD and glutathione synthetic enzymes [5]. MnSOD is encoded by genomic DNA and its dismutase function is fully activated in the mitochondria to detoxify free radical $\mathrm{O}_{2}{ }^{--}$generated by mitochondrial respiration [25]. In our study, decreases of PGC-1 $\alpha$ and MnSOD mRNA were found in hepatocytes and cardiomyocytes subjected to EHS. In contrast, salidroside 


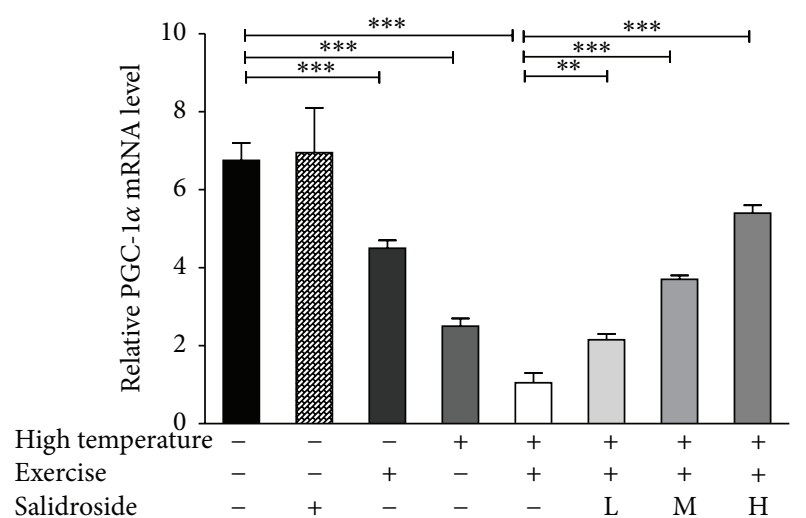

(a)

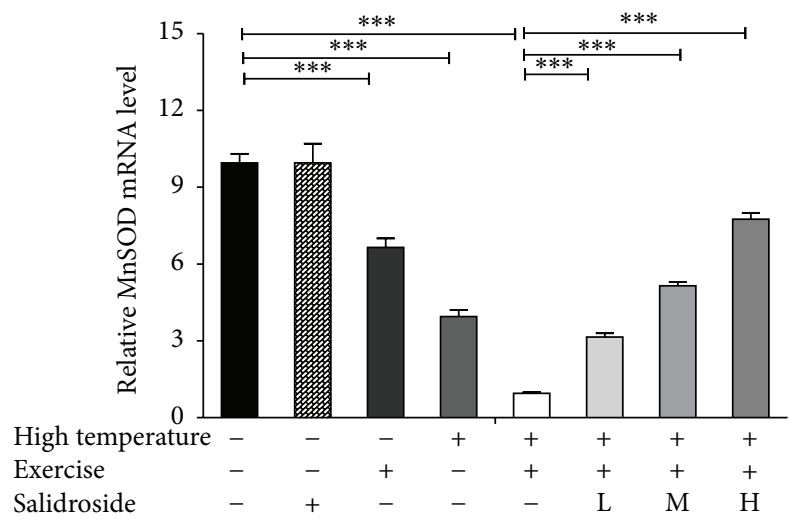

(c)

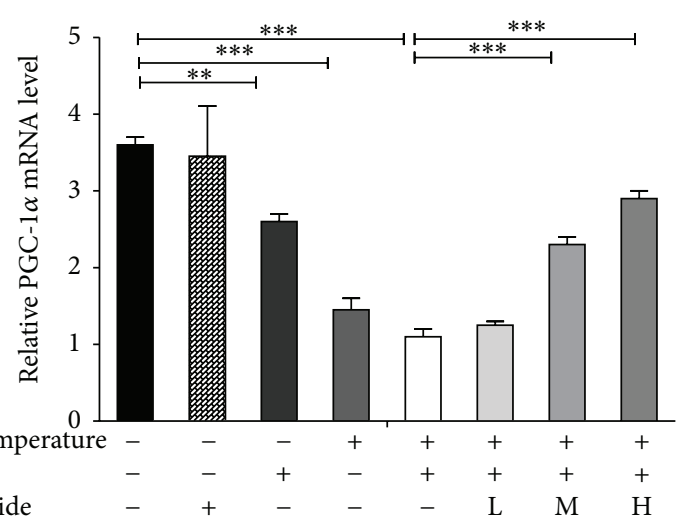

(b)

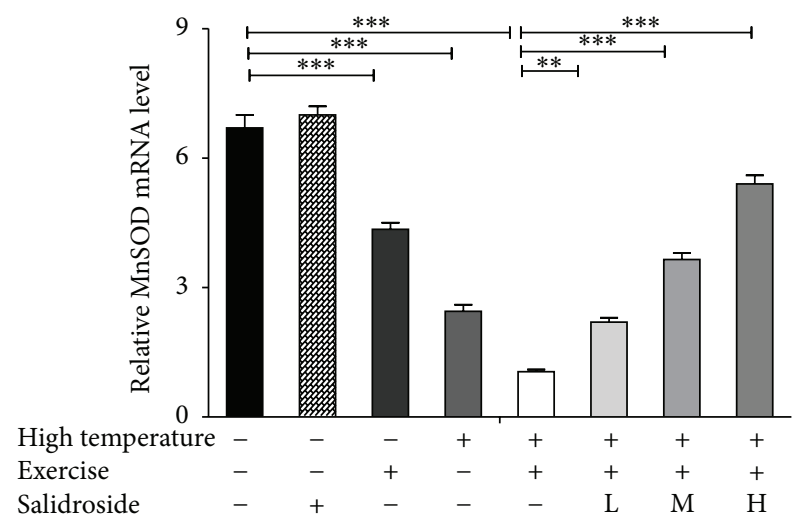

(d)

FIGURE 7: Salidroside reversed downregulation of PGC- $1 \alpha$ and MnSOD stimulated by EHS. (a) Relative PGC-1 $\alpha$ mRNA levels in hepatocytes in the control group, high temperature group, exercise group, EHS group, and EHS + salidroside group (20, 50, and 100 $\mu \mathrm{M}$ ). (b) Relative PGC- $1 \alpha$ mRNA levels in cardiomyocytes in distinct treatment groups. (c) Relative MnSOD mRNA levels in hepatocytes in distinct treatment groups. (d) Relative MnSOD mRNA levels in hepatocytes in distinct treatment groups. Results were expressed as the mean \pm SEM. $n=10$. ${ }^{* *} P<0.01$, and ${ }^{* * *} P<0.001$.

improved PGC-1 $\alpha$ and MnSOD mRNA and ameliorated such EHS-induced mitochondrial dysfunction.

In conclusion, we showed that salidroside exerts a protective effect against EHS-induced mitochondrial dysfunction and demonstrated that this occurs via inhibiting mitochondria swelling, preventing generation of ROS and MDA, and restoring $\triangle \Psi \mathrm{m}, \mathrm{RCR}$, free $\mathrm{Ca}^{2+}$ concentration, SOD, PGC$1 \alpha$, and MnSOD mRNA levels. More work in the future will be required to fully understand the beneficial role of salidroside against EHS and to further unfold the mystery of the mechanism involved.

\section{Disclosure}

Wei Zhang and Ming Peng are co-first authors.

\section{Conflict of Interests}

All authors declare that there is no conflict of interests regarding the publication of this paper.

\section{Authors' Contribution}

Wei Zhang and Ming Peng contributed equally to this work.

\section{Acknowledgment}

This work was supported by the Nanjing Military Region Science Foundation-funded major project (no. 10z033).

\section{References}

[1] V. M. S. Raj, A. Alladin, B. Pfeiffer et al., "Therapeutic plasma exchange in the treatment of exertional heat stroke and multiorgan failure," Pediatric Nephrology, vol. 28, no. 6, pp. 971-974, 2013.

[2] P. A. Ruell, D. Simar, J. D. Périard, S. Best, C. Caillaud, and M. W. Thompson, "Lymphocyte and monocyte Hsp72 responses to exercise in athletes with prior exertional heat illness," International Journal of Exercise Science: Conference Proceedings, vol. 10, no. 1, article 62, 2013.

[3] S.-H. Chen, M.-T. Lin, and C.-P. Chang, "Ischemic and oxidative damage to the hypothalamus may be responsible for heat 
stroke," Current Neuropharmacology, vol. 11, no. 2, pp. 129-140, 2013.

[4] J. Y. Li, Y. T. Qi, H. Liu et al., "Acute high-altitude hypoxic brain injury: identification of ten differential proteins," Neural Regeneration Research, vol. 8, no. 31, pp. 2932-2941, 2013.

[5] M.-C. Xu, H.-M. Shi, X.-F. Gao, and H. Wang, "Salidroside attenuates myocardial ischemia-reperfusion injury via PI3K/Akt signaling pathway," Journal of Asian Natural Products Research, vol. 15, no. 3, pp. 244-252, 2013.

[6] H. Zhong, H. Xin, L.-X. Wu, and Y.-N. Zhu, "Salidroside attenuates apoptosis in ischemic cardiomyocytes: a mechanism through a mitochondria-dependent pathway," Journal of Pharmacological Sciences, vol. 114, no. 4, pp. 399-408, 2010.

[7] M.-C. Xu, H.-M. Shi, H. Wang, and X.-F. Gao, "Salidroside protects against hydrogen peroxide-induced injury in HUVECs via the regulation of REDD1 and mTOR activation," Molecular Medicine Reports, vol. 8, no. 1, pp. 147-153, 2013.

[8] S. Yu, L. Liu, T. Wen et al., "Development and validation of a liquid chromatographic/electrospray ionization mass spectrometric method for the determination of salidroside in rat plasma: application to the pharmacokinetics study," Journal of Chromatography B: Analytical Technologies in the Biomedical and Life Sciences, vol. 861, no. 1, pp. 10-15, 2008.

[9] M. Yu, Y. Shi, X. Wei et al., "Depletion of mitochondrial DNA by ethidium bromide treatment inhibits the proliferation and tumorigenesis of T47D human breast cancer cells," Toxicology Letters, vol. 170, no. 1, pp. 83-93, 2007.

[10] A. M. Falchi, V. Sogos, F. Saba, M. Piras, T. Congiu, and M. Piludu, "Astrocytes shed large membrane vesicles that contain mitochondria, lipid droplets and ATP," Histochemistry and Cell Biology, vol. 139, no. 2, pp. 221-231, 2013.

[11] Y. Suzuki, H. Hasegawa, T. Tsuji et al., "Relationships of diverse apoptotic death process patterns to mitochondrial membrane potential $\left(\Delta \psi_{\mathrm{m}}\right)$ evaluated by three-parameter flow cytometric analysis," Cytotechnology, vol. 65, no. 1, pp. 59-70, 2013.

[12] A. D. Martin, A.-M. Joseph, T. M. Beaver et al., "Effect of intermittent phrenic nerve stimulation during cardiothoracic surgery on mitochondrial respiration in the human diaphragm," Critical Care Medicine, vol. 42, no. 2, pp. e152-e156, 2014.

[13] A. M. Petrov, A. A. Yakovleva, and A. L. Zefirov, "Role of membrane cholesterol in spontaneous exocytosis at frog neuromuscular synapses: ROS—calcium interplay," The Journal of Physiology, vol. 593, no. 2, pp. 4995-4500, 2014.

[14] A. Partyka, E. Łukaszewicz, and W. Nizański, "Effect of cryopreservation on sperm parameters, lipid peroxidation and antioxidant enzymes activity in fowl semen," Theriogenology, vol. 77, no. 8, pp. 1497-1504, 2012.

[15] A. A. Romanovsky and C. M. Blatteis, "Heat stroke: opioidmediated mechanisms," Journal of Applied Physiology, vol. 81, no. 6, pp. 2565-2570, 1996.

[16] C. Villeneuve, C. Guilbeau-Frugier, P. Sicard et al., "P53PGC- $1 \alpha$ pathway mediates oxidative mitochondrial damage and cardiomyocyte necrosis induced by monoamine oxidasea upregulation: role in chronic left ventricular dysfunction in mice," Antioxidants and Redox Signaling, vol. 18, no. 1, pp. 5-18, 2013.

[17] L. R. Leon and B. G. Helwig, "Heat stroke: role of the systemic inflammatory response," Journal of Applied Physiology, vol. 109, no. 6, pp. 1980-1988, 2010.

[18] C. Qin, S. Zhou, Y. Xiao, and L. Chen, "Erythropoietin enhances mitochondrial biogenesis in cardiomyocytes exposed to chronic hypoxia through Akt/eNOS signalling pathway," Cell Biology International, vol. 38, no. 3, pp. 335-342, 2014.

[19] V. N. Kotiadis, M. R. Duchen, and L. D. Osellame, "Mitochondrial quality control and communications with the nucleus are important in maintaining mitochondrial function and cell health," Biochimica et Biophysica Acta, vol. 1840, no. 4, pp. 12541265, 2014.

[20] M. Aghaei, F. Karami-Tehrani, M. Panjehpour, S. Salami, and F. Fallahian, "Adenosine induces cell-cycle arrest and apoptosis in androgen-dependent and-independent prostate cancer cell lines, LNcap-FGC-10, DU-145, and PC3," Prostate, vol. 72, no. 4, pp. 361-375, 2012.

[21] L. M. T. Canzoniero, A. Granzotto, D. M. Turetsky, D. W. Choi, L. L. Dugan, and S. L. Sensi, "nNOS(+) striatal neurons, a subpopulation spared in Huntington's Disease, possess functional NMDA receptors but fail to generate mitochondrial ROS in response to an excitotoxic challenge," Frontiers in Physiology, vol. 4, artilce 112, Article ID Article 112, 2013.

[22] L. Kastl, S. W. Sauer, T. Ruppert et al., “TNF-alpha mediates mitochondrial uncoupling and enhances ROS-dependent cell migration via NF-kappaB activation in liver cells," FEBS Letters, vol. 588, no. 1, pp. 175-183, 2014.

[23] S. K. Farahmand, F. Samini, M. Samini, and S. Samarghandian, "Safranal ameliorates antioxidant enzymes and suppresses lipid peroxidation and nitric oxide formation in aged male rat liver," Biogerontology, vol. 14, no. 1, pp. 63-71, 2013.

[24] A. Johri, A. Chandra, and M. F. Beal, "PGC-1 $\alpha$, mitochondrial dysfunction, and Huntington's disease," Free Radical Biology and Medicine, vol. 62, pp. 37-46, 2013.

[25] D. Candas and J. J. Li, "MnSOD in oxidative stress responsepotential regulation via mitochondrial protein influx," Antioxidants \& Redox Signaling, vol. 20, no. 10, pp. 1599-1617, 2014. 


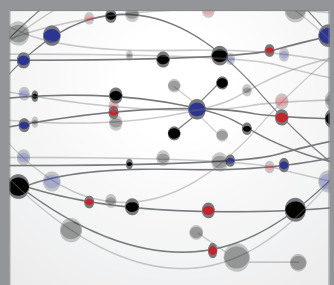

The Scientific World Journal
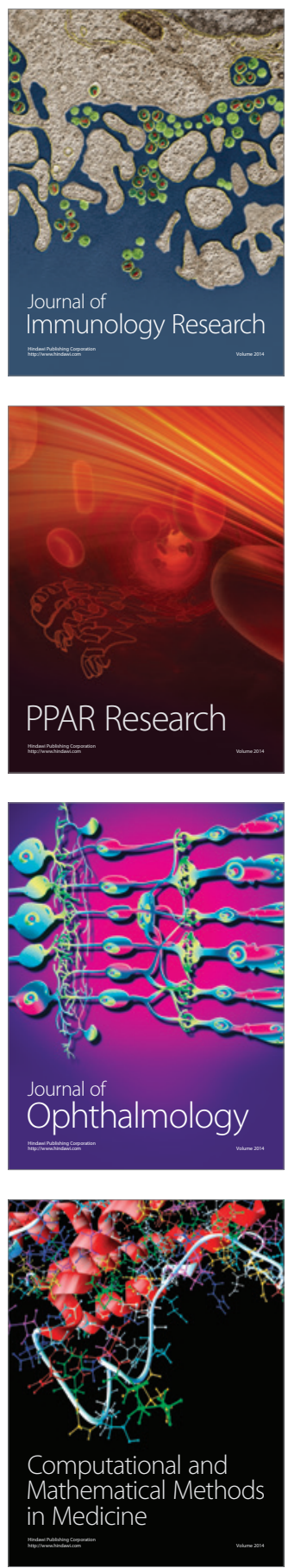

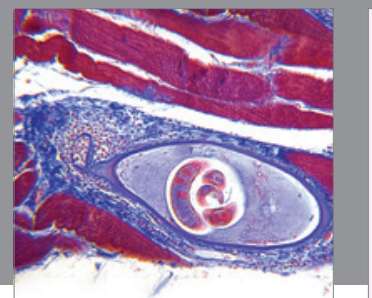

Gastroenterology

Research and Practice
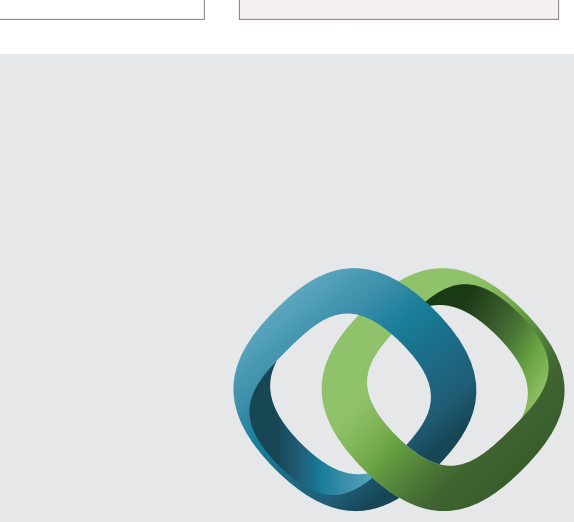

\section{Hindawi}

Submit your manuscripts at

http://www.hindawi.com
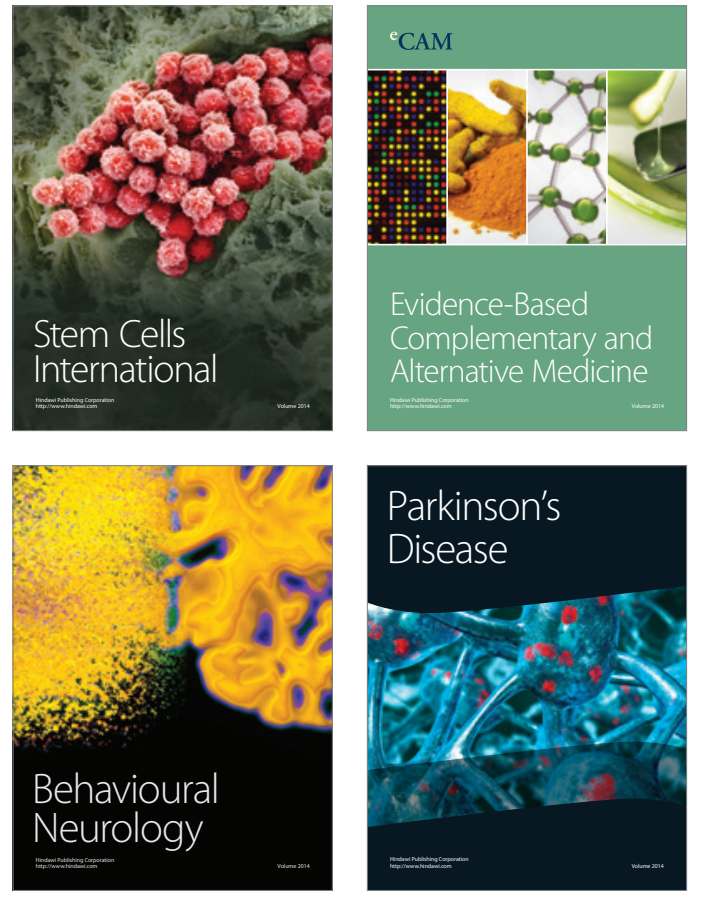
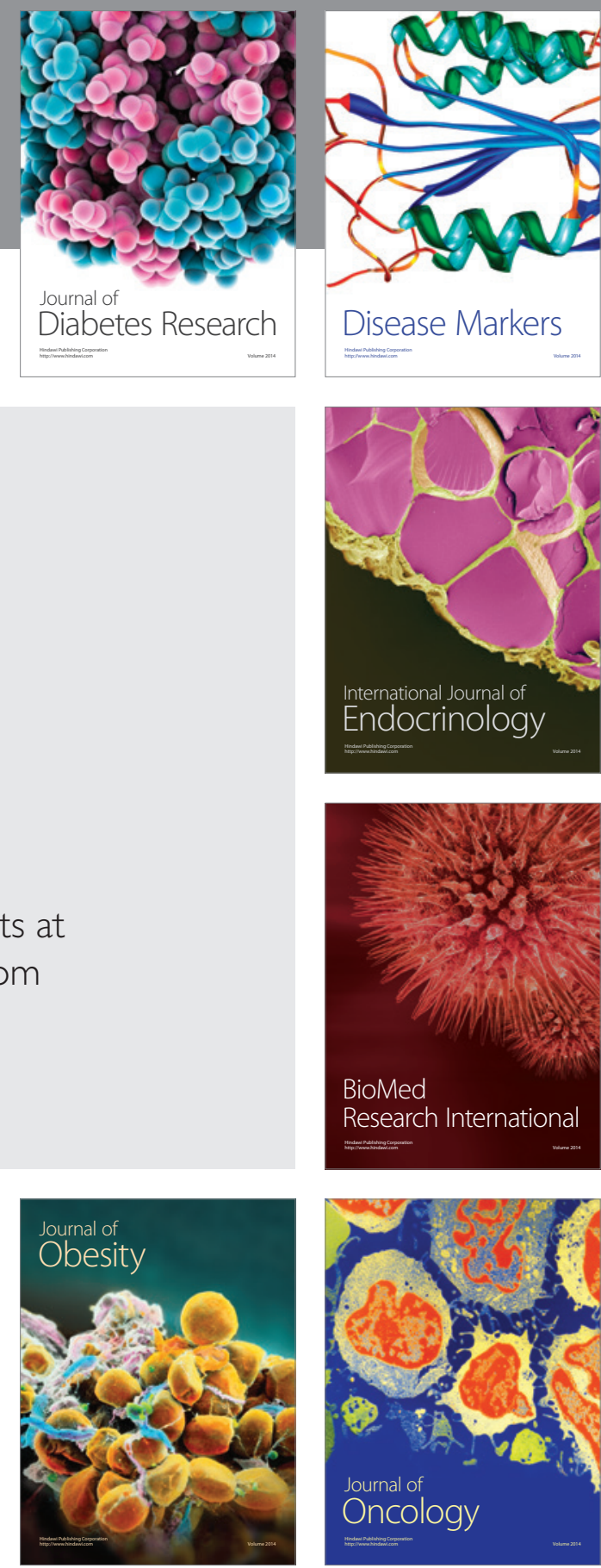

Disease Markers
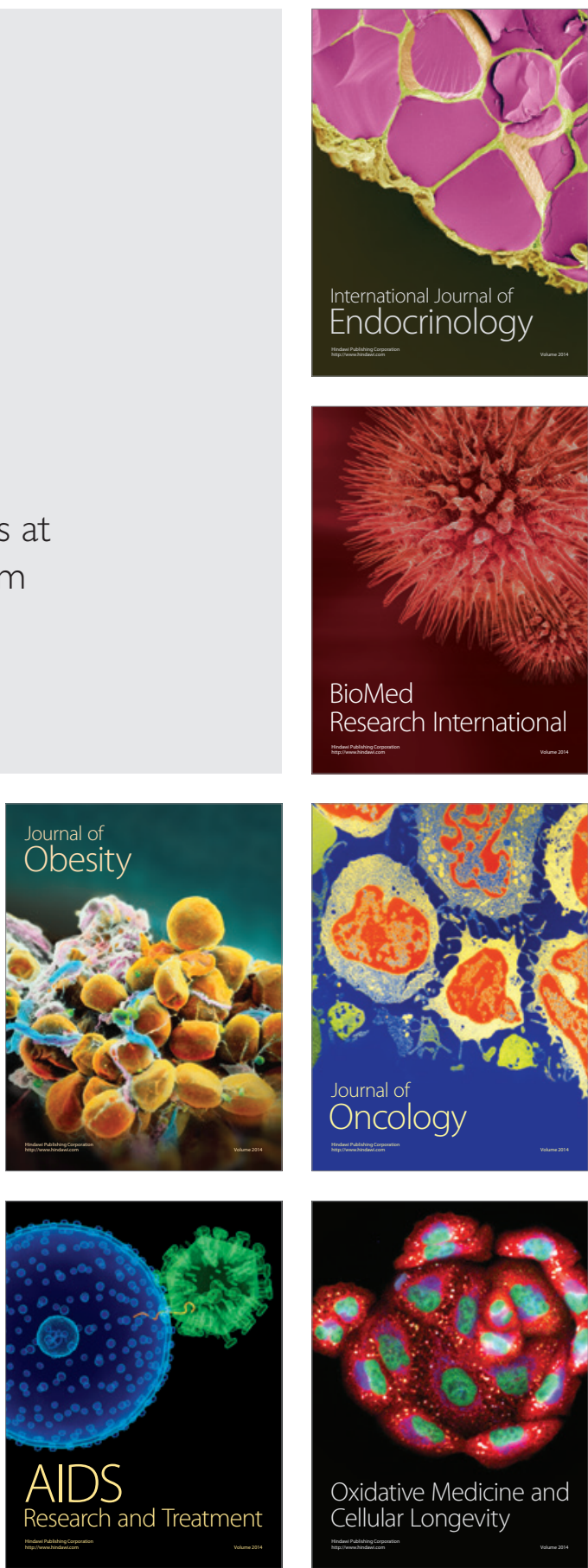